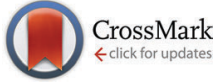

Cite this: Phys. Chem. Chem. Phys., 2015, 17, 2950

Received 30th September 2014, Accepted 9th December 2014

DOI: $10.1039 / c 4 c p 04427 d$

www.rsc.org/pccp

\section{Atomic force microscopy in biomaterials surface science}

\begin{abstract}
Fabio Variola*ab
Recent progress in surface science, nanotechnology and biophysics has cast new light on the correlation between the physicochemical properties of biomaterials and the resulting biological response. One experimental tool that promises to generate an increasingly more sophisticated knowledge of how proteins, cells and bacteria interact with nanostructured surfaces is the atomic force microscope (AFM). This unique instrument permits to close in on interfacial events at the scale at which they occur, the nanoscale. This perspective covers recent developments in the exploitation of the AFM, and suggests insights on future opportunities that can arise from the exploitation of this powerful technique.
\end{abstract}

\section{Introduction}

Important scientific discoveries have always followed closely the technological development of novel tools for observing natural and physical phenomena at various scales. For example, the evolution of the telescope, from Galileo's first astronomical observations to the latest evidence supporting the Big Bang theory, has propelled our knowledge of celestial events. At the other end of the scale, progress in microscopy techniques has revealed increasingly smaller features of matter, ranging from the finest structures of plants and biological tissues to single atoms. ${ }^{1}$ Among the various instruments that enabled to resolve what is invisible to the eye, the atomic force microscope (AFM) has rapidly emerged as one of the most efficient and flexible tools to probe materials at the micro- and nanoscales. ${ }^{2}$ One of the disciplines that has benefitted the most from this technique is undoubtedly biomaterials surface science, a research field that aims at elucidating the interactions between materials and the surrounding biological environment by synergistically integrating different branches of science such as nanotechnology, biology, chemistry and physics. In this context, the AFM has contributed significantly to determining that the activity of proteins, cells and bacteria is affected by the nanometric physicochemical features of surfaces. ${ }^{3-8}$ While the main focus of this perspective will be the nanoscale interactions between biological entities and synthetic biomaterials (i.e. abiotic materials intended to interact with biological systems), it should be noted that the AFM has also been advantageously exploited to probe the effects of the microscale surface properties on biological activity ${ }^{7,9,10}$ and

\footnotetext{
${ }^{a}$ Faculty of Engineering, Department of Mechanical Engineering, University of Ottawa, Ottawa, ON, K1N 6N5, Canada. E-mail: Fabio.variola@uottawa.ca

${ }^{b}$ Faculty of Science, Department of Physics, University of Ottawa, Ottawa, ON, K1N 6N5, Canada
}

to investigate naturally occurring biomaterials (e.g. bone, teeth, cartilages, collagen and biomembranes). ${ }^{11-15}$

Concisely, the AFM allows to image, measure (quantify), manipulate and sense matter at the nanometric level. From this first description, it readily transpires that this microscope is not simply an instrument to visualize small features, but instead a more powerful toolbox that offers additional remarkable capacities beyond imaging. These will be discussed below, aiming at providing the reader with a critical overview of selected applications of the AFM in biomaterials surface science.

\section{AFM basic principles}

The AFM consists of a sharp tip mounted at the end of a microfabricated flexible cantilever which is raster scanned across the sample's surface. When the tip is brought in close proximity to the sample surface, attractive and repulsive forces cause the deflection of the cantilever. This deflection is detected and processed as a function of the position on the $(x, y)$ plane in order to obtain topographical images. The AFM can operate in a variety of modes, including contact (the tip is in continuous contact with the surface), non-contact (the cantilever vibrates

Dr Fabio Variola received his MSc in Materials Engineering from the University of Trieste. He then completed a joint doctoral program between the Institut National de la Recherche Scientifique-Énergie, Matériaux et Télécommunications (INRS-ÉMT) and the Université de Montréal. Since 2010, Dr F. Variola has worked as an Assistant Professor in the Department of Mechanical Engineering/Physics at the University of Ottawa. His expertise conjugates materials engineering with surface science and nanotechnology. As an independent investigator, Dr F. Variola has focused on understanding the cellular and molecular mechanisms that dictate how mammalian cells and bacteria respond to biomaterials. 
and variations from its resonance frequency are used to generate images) and intermittent (the cantilever moves rapidly with a large oscillation between the repulsive and attractive forces). ${ }^{16}$ If, in place of scanning, the tip is placed at a fixed point and moved vertically towards the sample and then retracted (force-distance analysis), the deflection of the cantilever will provide information on the mechanical properties of the surface, such as stiffness (as the tip approaches and indents the sample) and adhesion forces (as the tip tries to disengage from the sample) (Section 3.4). ${ }^{16}$ For hard materials (e.g. metals, ceramics) the cantilever will simply approach the surface, jump into contact and then deflect (Fig. 1A, green line). For softer samples (e.g. polymers, cells) the curve will resemble the blue one in Fig. 1A. If adhesion forces generate between the tip and the surface, the cantilever will deflect downwards as it retracts away from the sample, jumping back to its initial position as the maximum pulling force of the cantilever exceeds the tip-sample adhesion (Fig. 1B, orange line). ${ }^{17}$ If a molecule or a single cell is coupled with the tip (Section 3.5), as the cantilever moves away from the sample, molecule-surface, molecule-molecule (e.g. ligand-receptor), cell-cell and cell-surface adhesion bonds will be progressively ruptured throughout a series of unbinding events until full detachment, generating the characteristics "jumps" in the forcedisplacement curve (Fig. 1C, orange curve). For a more exhaustive

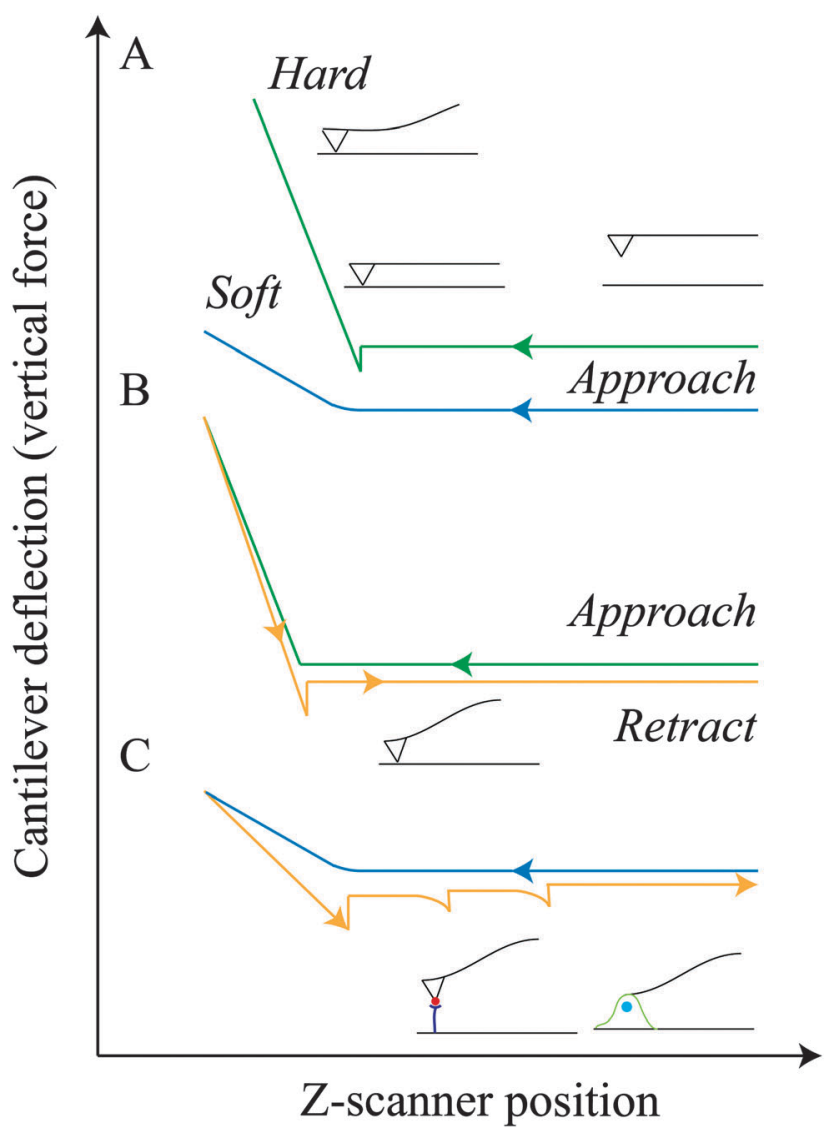

Fig. 1 (A) Typical force-distance curves for hard (green) and soft (blue) materials. (B) Adhesion on a hard surface. (C) Molecule-molecule and cell-surface detachment process with three unbinding events. coverage of the AFM basics, readers are invited to consult the comprehensive literature on the subject. ${ }^{16,17}$

\section{Major applications of atomic force microscopy}

\subsection{Imaging}

It is now well-known that nanotopography is one of the main parameters that governs protein adsorption onto biomaterials ${ }^{3}$ and dictates the interfacial interactions with cells and bacteria. ${ }^{4,8}$ Therefore, a precise 3-dimensional characterization of the nanometric features of surfaces is a fundamental step for understanding the biological response to nanostructured biomaterials. AFM conjugates high spatial resolution with experimental flexibility (i.e. it can be applied to diverse conductive and non-conductive materials, and requires little to no sample preparation), reasons that justify its widespread use not only to display the nanotopography of surfaces but also quantify their micro and nanoscale roughness. ${ }^{18-24}$ Despite its flexibility and advantages, this technique can be susceptible to artifacts that may lead to the erroneous interpretation of data. For instance, the actual profile of sharp vertical steps can be altered by the tip geometry and/or the radius of the tip itself. ${ }^{16}$ Similarly, the height/depth of porous/hollow structures (e.g. 3-dimensional scaffolds for tissue engineering and nanoporous platforms for drug-delivery applications), for which the full penetration of the tip is hampered by its geometry, may be underestimated. While these limitations can be overcome by using sharp and ultra-sharp tips, ${ }^{16,25}$ there is still a trade-off between the image quality and the mechanical strength of the tip itself. Sharp tips are in fact more prone to blunt and/or break, which can lead to additional artifacts. ${ }^{16}$

The AFM is not only capable of precisely resolving the nanotopography of surfaces, but also of mapping the spatial distribution of their physicochemical properties, such as, for instance, charge density and potential (i.e. Kelvin-probe force microscopy, a technique that detects and measures the electrostatic interactions between a conductive AFM tip and the substrate). ${ }^{26,27}$ In this context, the electrical properties of surfaces are critical factors in affecting cell-substrate interactions. ${ }^{28,29}$ It was in fact shown that nanotopographical surfaces influence charge density and electric field strength, and, in turn, the protein-mediated adhesion of cells. $^{28,30}$ In particular, it was proposed that positively charged proteins (or proteins with positively charged domains) are responsible for dictating the subsequent attachment of negatively charged cells onto a negatively charged nanorough surface. ${ }^{29}$ While the majority of these studies employed computational methods to elucidate proteins and cell behavior on such surfaces, only a few exploited experimental approaches to validate the proposed analytical models and mechanisms. ${ }^{31}$ To this end, the capacity of AFM to visualize nanotopographical features and correlate them to surface charge density and potential ${ }^{26,27}$ will offer interesting new opportunities to consolidate the present knowledge of the relationships between the nanotopography and electrical characteristics of biomaterials, and ultimately determine how these influence proteins, cells and bacteria functions. 


\subsection{AFM and other imaging techniques}

In general, compared to other techniques such as standard Scanning (SEM) and Transmission Electron (TEM) microscopy, the AFM not only offers the capacity to visualize nanometric features in two and three dimensions (for significantly smaller areas though, when compared to SEM) and to map the physicochemical characteristics of surfaces, but it also advantageously permits to do so in air, vacuum and especially in liquid environments. These characteristics have been fundamental to image static and dynamic events, such as the adsorption of proteins and DNA, ${ }^{32-36}$ as well as the structural and molecular components of living cells and bacteria (Fig. 2), ${ }^{37-44}$ while providing minimal perturbation of the native characteristics of biological samples and avoiding artifacts associated with drying, including the creation of artefactual structures (e.g. dendritic patterns resulting from the crystallization of the buffer). ${ }^{16}$ In this context, for cells and bacteria imaging, chemical immobilization is often required to anchor them onto substrates in order to prevent their movement during imaging (common methods include fixation with glutaraldehyde and adsorption onto surfaces pre-coated with polymers, among others). ${ }^{39,45}$ This procedure may however alter the nature of interfacial interactions, ${ }^{46}$ thereby posing a challenge for studies aimed at assessing the direct effects of specific surface cues on the biological response. Standard fixation procedures that involve proteins crosslinking can also perturb the natural properties of cell wall architecture, ${ }^{46}$ and, in turn, may affect nanomechanical measurements (Section 3.4). The attachment methods that result in the nearest to natural state for cells are those that do not involve any chemical treatment, ${ }^{45}$ such as physical entrapment in porous membranes, confinement in micro-wells and electrostatic interactions. ${ }^{39}$ Noteworthy, immobilization protocols for live imaging may not be always necessary if cells adhere onto nanostructured biomaterials. These have in fact shown to promote a firmer cellular adhesion in physiological conditions by direct physical cueing, ${ }^{47}$

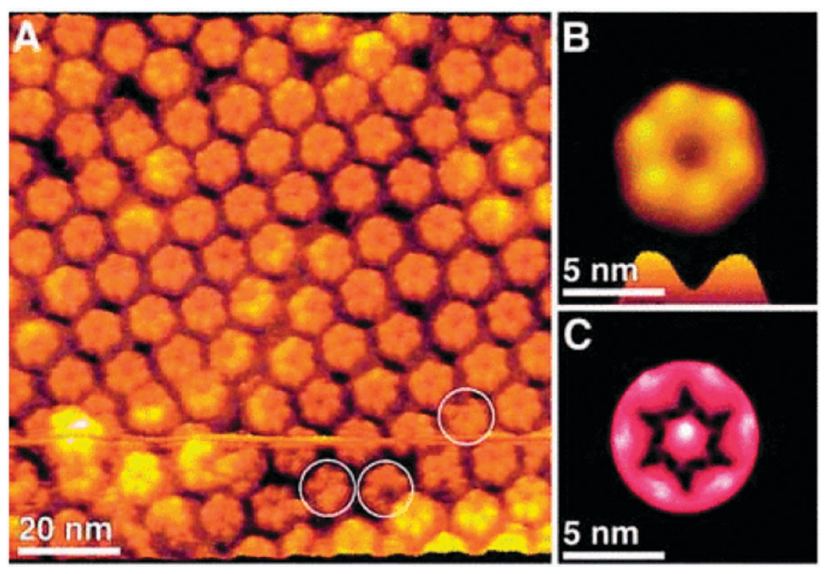

Fig. 2 (A) AFM images of the arrangement of connexons (hexameric halfchannels that bridge the extracellular space between adjacent plasma membranes) in gap junctions plaques. (B) Top and side view of a single connexon. (C) Enhanced structural details of a single connexon. Reprinted with permission from ref. 37. Copyright 2002 American Chemical Society. and thus promise to provide the required adhesion strength for imaging without any external fixation protocol. In general, the AFM procedure for imaging living cells should be fast and only rely on very small interaction forces to prevent cells from moving, detaching and/or being damaged. In this context, intermittent AFM-based approaches permit to reduce the imaging time, minimizing the risk of movement of the cell during the scan. ${ }^{46,48}$ In particular, Digital Pulsed Force mode (DPFM) imparts sinusoidal movement to the cantilever and modulates its oscillation at high frequency to make consecutive tip indentations on the surface, ultimately yielding faster scanning speeds while exerting small and controlled interaction forces. ${ }^{48,49}$ These and similar results (Section 3.4) demonstrate that effective AFM-based approaches to image and assess the structural properties of living cells and bacteria are readily available, and undoubtedly offer new opportunities to investigate cellular functions and interactions with surfaces with minimal perturbation.

\subsection{Chemical force microscopy}

The already impressive imaging capacities have been even further expanded by integrating the AFM's spatial resolution and sensitivity with chemical functionalization, a set of strategies to immobilize molecular agents onto the cantilever's tip. The possibility of tailoring the AFM probe with chemical groups and individual molecules, coupled with the ability to carry out measurements under near-physiological conditions, made this approach ideal for biological applications. ${ }^{50}$ To date, chemical force microscopy (CFM) has been successfully exploited to visualize the distribution of molecules in surface-adsorbed protein layers $^{51}$ and map the supramolecular organization of cellular membranes. ${ }^{52-55}$ These abilities also extend to the investigation of bacteria, and have permitted to visualize the structure and properties (e.g. hydrophobicity) of wall components (Fig. 3). ${ }^{39,56,57}$ Because of the abundance and flexibility of chemical strategies that can be tailored to precisely target selectively chosen features, ${ }^{58}$ tip functionalization and force imaging hold the potential not only to display, but also quantify (Section 3.5), a wide variety of relevant interfacial phenomena, such as the spatial distribution of specific binding sites on surfaces and its effects on the activity of adhering cells.

\subsection{Nanomechanical measurements}

In parallel with imaging capacities, one of the distinctive features that has propelled the use AFM in nanosciences is undoubtedly its capability to quantify physicochemical properties of materials and biological entities. To date, AFM analysis has yielded a deeper understanding of the nanoscale mechanical properties of materials, proteins, cells and bacteria, supporting standard characterization and microscopy techniques (e.g. optical and fluorescence microscopy) with quantitative data. Advances that stemmed from such approach will be presented in more detail below, providing further evidence of the power of the AFM as a multifunctional nanoscale tool.

A growing number of studies has been exploring the role of micro- and nano-level mechanics on the cellular response to surfaces. ${ }^{59-62}$ For example, it is now well known that adhering 

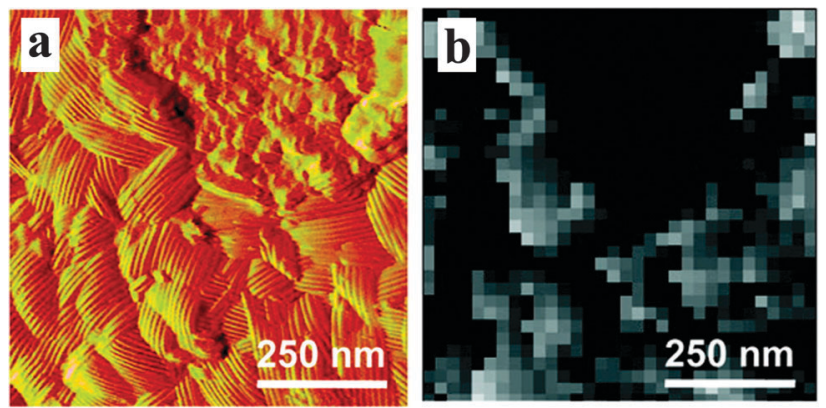

Fig. 3 (a) AFM topographic image of the nanoscale structure of a microorganism (Aspergillus fumigatus) and (b) chemical force image of its hydrophobic domains. Reprinted with permission from ref. 56. Copyright 2007 American Chemical Society.

cells sense and respond to the substrate's stiffness. ${ }^{63}$ In this context, the AFM's capacity to quantify the stiffness and the hardness of natural and synthetic materials has provided important new resources for materials scientists interested in the connections between the nanomechanical characteristics of surfaces and the physicochemical properties of bulk materials. ${ }^{49,64}$ In fact, compared to standard techniques (e.g. tensile testing, microhardness, etc.), the AFM distinctively offers a non-destructive way to probe multiple properties at once (i.e. stiffness, maximum indentation, indentation at maximum force, hysteresis, hardness, compliance, adhesion force, detachment distance, detachment energy, Young's modulus ${ }^{49}$ with a nanometric spatial resolution, and to simultaneously overlap them to the morphological images obtained with one of the modes outlined in Section 2.

In parallel, biologists and biophysicists have capitalized on force-distance measurements (Section 2) to close in on the nanomechanical properties of cells and bacteria such as Young's modulus and deformation, ${ }^{46,65,66}$ and determined how these vary during adaptation to the extracellular environment and/or to external physicochemical stimuli. ${ }^{62,65,67-69}$ A particular attention has been given to assessing how mammalian cells translate mechanical cues into cytoskeletal changes and biochemical signaling, and how the mechanical properties of cells are involved in regulating cellular functions (mechanosensing, mechanotransduction, and mechanoresponse). ${ }^{69}$ In this context, intermittent techniques allowed to map the mechanical properties of cells and bacteria, offering the distinctive advantage of providing their spatial distribution within single cells. For example, the mapping and time evolution of the stiffness of cancer cells were successfully imaged by DPFM (Section 3.2). ${ }^{48}$ In parallel, a recent study exploited a similar quantitative AFM procedure that provides precise and continuous control on the acquisition velocity and vertical forces to investigate living bacteria. ${ }^{46}$ The bacterial suspension was gently deposited on a glass substrate and force distant curves were collected for every pixel of AFM images, simultaneously generating topography and stiffness maps without the need of any external immobilization step (Fig. 4).

While these studies have undoubtedly provided a great contribution in advancing knowledge in their respective fields, they appear to have progressed along two sets of parallel lines,

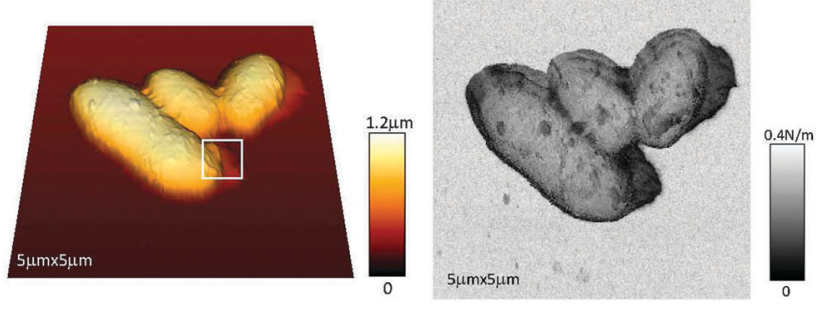

Fig. 4 AFM height (left) and stiffness (right) images of bacteria (Rhodococcus wratislaviensis) in their physiological medium. Reprinted with permission from ref. 46. Copyright 2013, PLoS One.

focused on either the nanomechanical properties of materials or on the biomechanics of biological entities. Ultimately, these two lines should converge towards the investigation of the mechanoresponse to the local physical/mechanical cueing exerted by nanostructures. In fact, it could be conceived that nanoscale surface features engender a precise nanomechanical environment capable of provoking localized cell-distortions, inducing biomechanical changes in cytoskeletal organization and cellular shape. ${ }^{7}$ Although further refinements are still needed, these phenomena can all be characterized by the AFM, which thus promises to support previous ${ }^{63}$ and ongoing efforts to bridge the gap between substrate and cellular mechanics. In this context, although the AFM has been successfully employed to quantify the stiffness of bacterial membrane,${ }^{46}$ its application to study how bacteria respond to nanostructured surfaces ${ }^{70}$ from a nanomechanical viewpoint is still lagging behind. Once addressed, the generated knowledge is poised to bring new evidence to elucidate the mechanisms involved during the interactions between bacteria and nanostructured antibacterial surfaces. ${ }^{71-73}$

\subsection{Force spectroscopy}

In addition to force imaging, tip functionalization has also permitted to quantify, with unparalleled spatial $(\sim 1 \mathrm{~nm})$ and force (in the order of piconewtons) resolution, a remarkable collection of molecular, cellular and bacterial properties and functions (Force spectroscopy). ${ }^{53}$ In the force spectroscopy technique, the AFM tip is functionalized with chemical groups (i.e. chemical force spectroscopy-CFS), biomolecules, proteins and viruses (i.e. single-molecule force spectroscopy-SMFS), as well as with single living cells (i.e. single-cell force spectroscopySCFS), and interactive forces acting on the functionalized tip when scanned across a sample are measured. ${ }^{39}$ This approach has provided direct access to a variety of inter- and intramolecular phenomena and cellular events otherwise inaccessible with other techniques. For example, single-molecule force spectroscopy quantified via force-distance curves protein unraveling, folding and unfolding mechanisms, receptor-ligand and ligandbinding interactions..$^{53,74,75}$ Research aiming at characterizing protein-biomaterials interactions can capitalize on these and similar studies ${ }^{76}$ and determine how nanometric surface cues affect parameters of protein adsorption such as adhesion and unbinding forces. The capacity of SMFS to probe molecular events can also be exploited to investigate the interactions and 
the dynamics of a variety of cell-surface-associated proteins. ${ }^{77}$ This also extends to bacteria research, where this technique has enabled a better understanding of the molecular mechanisms underlying the adhesive and mechanical properties of polysaccharides and proteins on live cells. ${ }^{78,79}$ Based on these studies, researchers are offered the opportunity to exploit SMFS to dissect out specific aspects of the molecular bases of cellular and bacteria adhesion processes on nanostructured biomaterials, to determine, for instance, whether the physicochemical cueing exerted by surface cues also affects the supramolecular architecture of adhering cells.

A field that will particularly benefit from advancements in SMFS is drug-delivery, particularly its branch that employs nanostructured biomaterials for localized and controlled elution of antibiotics, growth factors and viruses. ${ }^{80-82}$ In fact, the type and strength of the specific interactions established by bioactive molecules with surfaces are expected to impact their tendency to desorb, ultimately dictating the overall release profile. ${ }^{18}$ While the ability of the AFM to quantify the strength of adhesion forces can nonetheless provide important guidance for assessing the quality of molecule-surface interactions, care should be taken to relate the measured forces to the binding affinity, which was shown to depend to a large extend on the experimental parameters (e.g. contact time). ${ }^{76}$ In this context, various groups have been exploring the controlled delivery of viral vectors to overcome some of the current challenges in gene therapy and tissue engineering by improving site targeting, reducing inflammatory and immune responses, enhancing transduction and extending the duration of gene expression. ${ }^{82}$ Recently, strategies for specific vector-biomaterial binding based on the interactions of functional groups on viral capsid and functionalized surfaces (e.g. with antiviral antibodies) have been developed. ${ }^{82}$ The main goal is devising flexible approaches that permit to tailor the release and delivery to meet specific therapeutic needs by tuning the vector affinity for the surface. To this end, researchers can capitalize on previous work which exploited the AFM to determine how the structural and nanomechanical properties of viruses (e.g. stiffness of the viral shell), ${ }^{82-87}$ as well as their binding strength with materials, quantified by SMFS for instance, are affected by the physiochemical environment of surfaces. The AFM thus promises to become an essential tool also in the field of substrate-mediated viral gene delivery that will permit to make the correlation between the nature and quality of the initial virus-surface interactions and the resulting elution profiles. This will provide the key for optimizing vector-presenting substrates and design better performing drug-eluting platforms capable of retaining and progressively releasing the virus for cellular internalization. In addition, tuning surface properties to control vector-material interactions is also expected to unlock new strategies to preserve vector activity and avoid recognition by the immune system. ${ }^{82}$

At the cellular level, single cells can be ingeniously fixed onto the cantilever, becoming the probe for the dynamic quantification of cell-substrate interactions (SCFS). ${ }^{88,89}$ Parameters such as maximum detachment force and work for the entire cells as well as a number of detachment events of single cellular tethers have already been successfully quantified (Fig. 5). ${ }^{88,89}$ It should be noted that this technique still faces experimental challenges and limitations. ${ }^{88}$ For example, immobilization strategies have to ensure a firm attachment of individual cells onto the cantilever in ways that the cantilever-cell adhesion strength will be greater than the cell-substrate interactions to prevent the cell from adhering onto the substrate during the retraction of the tip. It follows that nanostructured materials that promote a strong cellular adhesion may cause the detachment of the cell from the cantilever. In addition, SCFS experiments are time consuming because only one cell can be characterized at a time. For statistical reasons, many detachment-force-distance curves must be recorded, which limits the length of the contact times that can reasonably be assayed, often restricting the analysis to short contact times (from milliseconds to about 20 minutes) ${ }^{88}$ Despite these challenges, SCFS nonetheless provides unique insight into
A

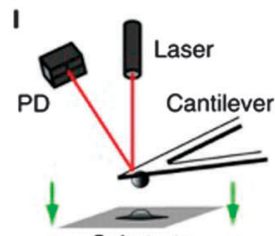

Substrate

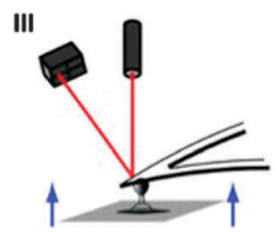

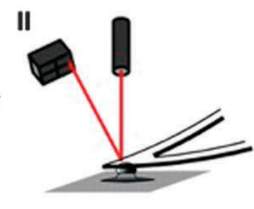

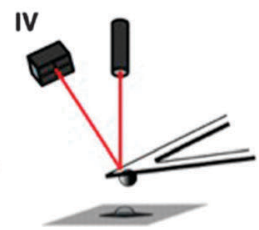

B

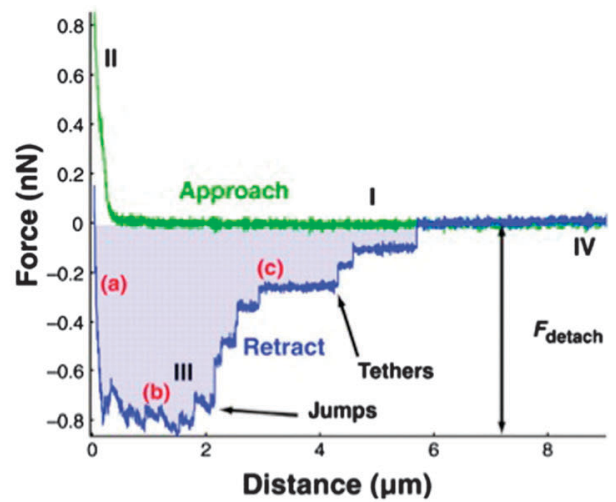

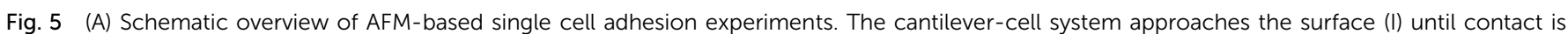

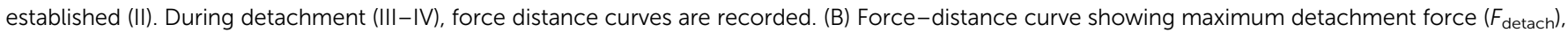

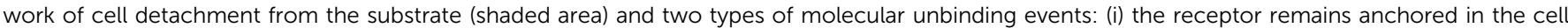

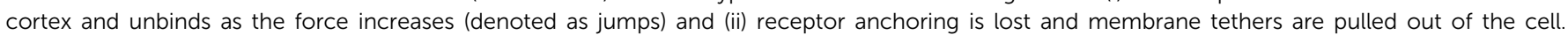
Reprinted with permission from ref. 88. Copyright 2008, Company of Biologists. 
the forces, energetics and kinetics of cell adhesion processes onto surfaces. ${ }^{88}$ In this context, it will be interesting to move the focus from whole cells to more specialized cellular components, and complement existing techniques (e.g. optical tweezers) ${ }^{90}$ with AFMbased approaches to investigate the local properties of filopodia emitted by cells adhering on nanostructured surfaces. A better understanding of the relationship of filopodia with surface features at their functional sensing scale, that is the nanoscale, will be in fact highly relevant for exploiting nanotopographical surfaces to stimulate (i.e. regeneration) and even slowdown (i.e. cellular hyperplasia, cancer cells) cell activity.

While single cell force spectroscopy has been implemented for studies involving mammalian cells, its application to bacterial research has not evolved as rapidly, mainly as a consequence of difficulties in immobilizing bacteria onto cantilevers. However, a recent article has reported the use of a cell adhesive to attach a single bacterium onto the cantilever and probe its interactions with different surfaces and other bacteria, ${ }^{91}$ thereby opening the window on additional possibilities to exploit the AFM to comprehend the mechanisms involved in the bacterial attachment to nanostructured surfaces.

To conclude, AFM force imaging and spectroscopy have permitted to achieve, through quantitative measurements of an unparalleled resolution, a deeper understanding of numerous molecular processes that underlay proteins, cells and bacteria activities. These techniques are not solely limited to biomaterials surface science, but their potential extend to other fields of science, ranging from drug delivery to medicine, where the capacity to quantify structures and interactions at the molecular level can contribute, for instance, to devise better performing pharmacologic strategies and elucidate the mechanisms of specific diseases.

\subsection{Scanning probe lithography}

In a further extension of AFM-based methods and techniques, the distinctive capacity to manipulate single molecules and detect their interactions has permitted researchers to explore additional applications in nanosciences, ultimately developing protocols and practices that harnessed the power of the AFM probe and transformed it into a multifunctional molecular toolbox. ${ }^{53}$ One of the most relevant techniques that stemmed from such a development falls under the umbrella of scanning probe lithography, a set of nanofabrication strategies in which the tip is used to directly pattern substrates with a variety of molecular "inks". ${ }^{16}$ Among these, dip-pen nanolithography has shown promising applications in biomaterials surface science. ${ }^{92}$ In this method, selected molecules are adsorbed by capillarity onto the AFM tip, which is successively used in contact-mode to transfer pre-determined molecular patterns to the surface with a resolution of $30-100 \mathrm{~nm} .{ }^{16}$ For instance, microarrays composed by DNA-directed protein immobilization have been precisely patterned and utilized to investigate the recruitment of transmembrane receptors in living cells (Fig. 6). ${ }^{93}$ This technique has been optimized on atomically-flat and contaminant-free surfaces (e.g. gold and silicon), and it can now count on a dramatically improved patterning efficiency and flexibility in depositing a)

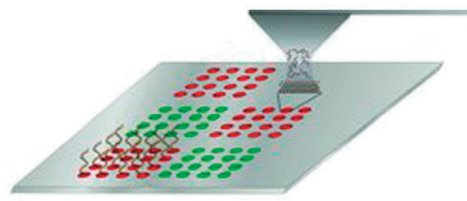

b)
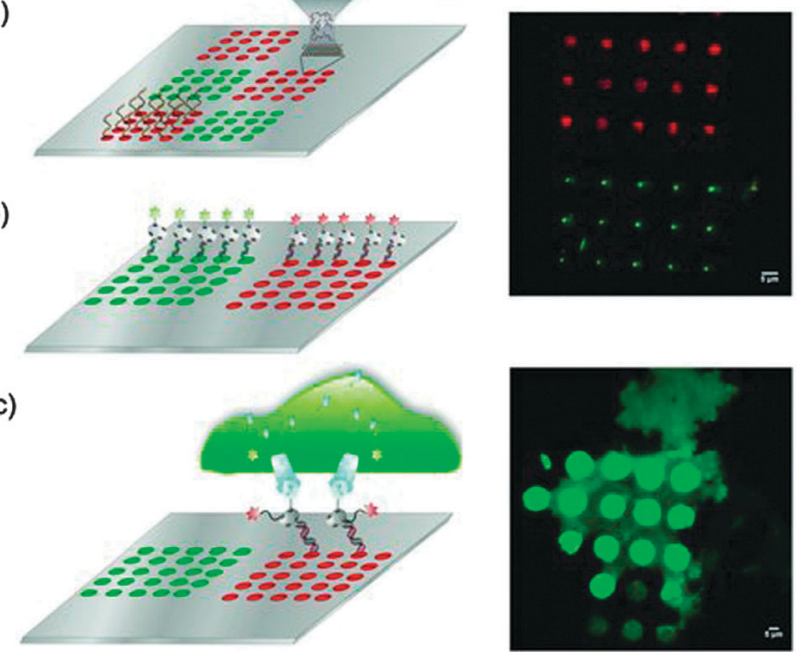

Fig. 6 Schematic representation of the generation of microarrays for DNAdirected protein immobilization for the recruitment of transmembrane receptors (left, from top to bottom). Live-cell arrays (top right) and expression of transmembrane protein in breast cancer cells membrane (bottom right). Reprinted with permission from ref. 93. Copyright 2013, Wiley.

different ink molecules. ${ }^{92}$ However, its translation to medically relevant materials and applications may still be far, mainly because of the non atomic planarity of most biomaterials and the practical need to pattern areas whose dimensions lay beyond the range of this technique. Nonetheless, the capacity of creating specific molecular patters will undoubtedly benefit more fundamental studies in biomaterials surface science, focused, for example, on furthering the investigation of cellular response to specific chemical cues (e.g. chemotaxis) ${ }^{94}$ and/or on the validation of functional molecular nanostructures. ${ }^{95,96}$

\subsection{Cantilever-based sensors}

In parallel with these capacities, the AFM has also been employed to detect the deflection and/or resonance shift of cantilever arrays used as micro and nanomechanical sensor for diagnostic and detection purposes. ${ }^{97-100}$ In this context, researchers have demonstrated that silicon-based cantilevers can be functionalized with thin metallic (e.g. gold) and polymeric (e.g. polystyrene, PMMA) films to detect a variety of biological and chemical species. ${ }^{101-103}$ The knowledge acquired so far in the field may permit to change the identity of the deposited materials and explore functionalization strategies to create thin films of biocompatible materials with controlled properties directly on cantilever arrays, which could then be used to study protein adsorption, ultimately complementing techniques such as surface plasmon resonance (SPR, i.e. an optical technique sensitive to the changes in refractive index that occur when proteins and other molecules accumulate onto a gold surface) and quartz crystal microbalance (QCM, i.e. a physical technique that detects changes in the resonance frequency of an electrically driven quartz crystal with changes in mass due to molecule adsorption). ${ }^{104,105}$ 


\section{Outlook}

The recent history of microscopy has witnessed a more systematic approach to the development and exploitation of novel strategies and protocols for the characterization of biomaterials and interfacial biological events at the micro- and nanoscales. In this context, the AFM has undoubtedly been a fundamental resource in biomaterials surface science to date, and will still play a major role in the future. In particular, the optimization of current tip functionalization approaches and the development of novel strategies for CFM, CFS, SMFS and SCFS will extend the breath of its applications and the specificity of the results, offering new opportunities to close in on still unknown aspects of how proteins, bacteria and cells sense and respond to nanostructured surfaces.

However, there is still a number of experimental limitations that will need attention to take the technique to a higher level and further enhance its impact. For instance, structural AFM measurements on living cells/bacteria still pose experimental challenges, not only in regard to immobilization protocols (Section 3.2), but also in relation to sample damaging caused by forces exerted by the tip, imaging artifacts and interpretation of quantitative results. In addition, the time currently required to record a high-resolution image is much longer than the timescale of most biological processes. ${ }^{69}$ To address this need, the development and optimization of fast scanning AFMs and acquisition procedures (e.g. DPFM) for high-speed topographic imaging now permit to image the physical, chemical and biological properties of samples at high speed and resolution. ${ }^{68,69,106}$ Applied to biomaterials surface science, these new advances hold the potential to yield a dynamic description of the events at the molecular scale that affect and control cell-surface interactions.

In such a technological evolution towards more advanced and effective analyses, the AFM has joined a more general trend in microscopy which aims at multimodal imaging capacities and correlative analyses, becoming an instrument that can seamlessly integrate complementary characterization methods. This evolution has ultimately permitted to the AFM to achieve a synergy that extends beyond the capacities and potential of the constituent techniques used independently.

Standard and high-resolution fluorescence microscopy as well as Raman imaging and spectroscopy ${ }^{107-109}$ can all be combined within the AFM setup. Such advances now permit to achieving highresolution images that simultaneously display the 3-dimensional topography of the cytoskeleton and its stiffness, and correlate them to its structural elements, such as the distribution of actin filaments visualized by standard confocal microscopy or by stimulated emission depletion microscopy (STED), a super high resolution technique that breaks the resolution limit of standard fluorescence microscopy (Fig. 7) ${ }^{110,111}$ This integrated approach is poised to unveil the connections between the cell nanomechanical properties and the organization of actin fibers and microtubules in the cellular body and its extensions, ultimately addressing, for instance, how the structural and mechanical parameters of cells and bacteria are affected by the physicochemical environment of surfaces. Similarly, when
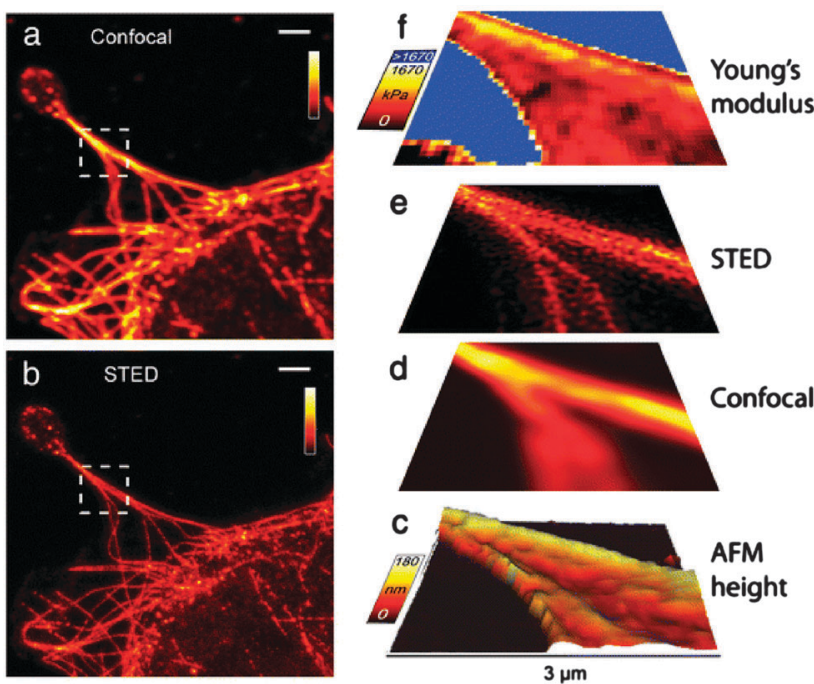

Fig. 7 (a) Confocal image and (b) STED image [both raw data], (c) 3D rendered view extracted from AFM force curves; deconvolved (d) confocal image and (e) STED image, (f) elasticity map calculated from AFM force curves of fibroblastic cells. Scale bars in (a) and (b): $2 \mu \mathrm{m}$, axes bars in (c)-(f): $3 \mu \mathrm{m}$. Reprinted with permission from ref. 111. Copyright 2012, SpringerOpen.

the AFM is coupled with a near-field scanning optical microscope (SNOM/NSOM), morphological and immunofluorescent information, such as the localization of molecular nanoclusters in cell membranes and protrusions (Fig. 8), can be overlapped. ${ }^{112-115}$ This promises to shed new light on the characteristics and variations of the cellular supramolecular architecture as a function of cellsubstrate and cell-cell interactions on nanostructured biomaterials. The integration of Raman spectroscopy and imaging offers the distinctive advantage to carry out label-free chemical analyses of surfaces and adhering cells and bacteria, ${ }^{116,117}$ and successively correlate them to the nanotopography of the underlying nanostructures. Raman spectroscopy will also permit to probe, in a non-destructive manner, the crystallinity and even local stress fields of biomaterials, ${ }^{118-121}$ parameters known to affect cell and bacteria response., ${ }^{7,93}$ It should be noted that the spatial resolution of standard Raman imaging is dictated by the optical objective and the laser wavelength, and can achieve at best a few hundreds of nanometers, thus considerably above that of the AFM. Such a limitation can however be overcome by tip-enhanced Raman spectroscopy (TERS), a technique that significantly increases the resolution of Raman imaging and

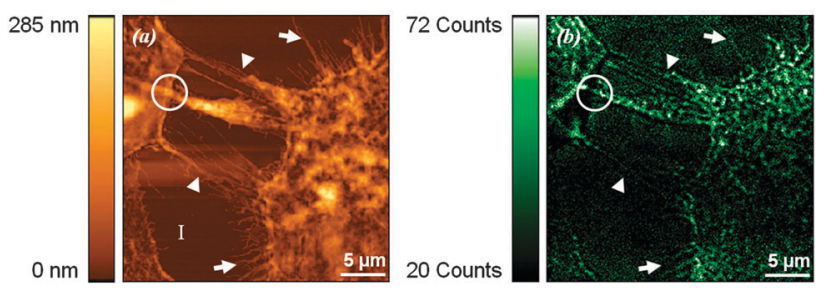

Fig. 8 Topographic (left) and fluorescent (right) SNOM images of human epithelial cells initiating cell-cell contact. Protein (E-cadherin) clusters location is revealed. Reprinted with permission from ref. 113. Copyright 2012, Elsevier. 
spectroscopy to a few nanometers. ${ }^{122}$ TERS have been used to investigate biomolecules, such as DNA bundles, ${ }^{123}$ and probe materials surfaces and biological samples at the nanoscale, ${ }^{124-128}$ thereby becoming a very promising technique for current and future studies in various disciplines.

The development of AFM-based integrated systems and, as a matter of fact, complementary multimodal instruments (e.g. Raman-SEM), is paving the way for a novel approach to applied research, in which the synergistic combination of different experimental techniques increasingly generates more efficient and flexible tools for the nanoscale characterization of synthetic and biological materials. The technical evolution that has propelled such correlative approach to science will also give new strength to biomaterials surface science, a field in which the AFM will continue to affirm its role as a unique resource.

\section{Acknowledgements}

F.V. thanks Drs Antonio Nanci and Shan Zou for critical reading of the manuscript and acknowledges funding from the Canada Foundation for Innovation (CFI), the Ontario Ministry of Research and Innovation, and the Natural Sciences and Engineering Research Council of Canada (NSERC).

\section{References}

1 C. Toumey, Nat. Nanotechnol., 2012, 7, 205-206.

2 P. Eaton and P. West, Atomic Force Microscopy, 2010.

3 M. S. Lord, M. Foss and F. Besenbacher, Nano Today, 2010, 5, 66-78.

4 M. J. Biggs, R. G. Richards and M. J. Dalby, Nanomedicine, 2010, 6, 619-633.

5 R. E. McMahon, L. Wang, R. Skoracki and A. B. Mathur, J. Biomed. Mater. Res., Part B, 2013, 101, 387-397.

6 L.-C. Cheng, X. Jiang, J. Wang, C. Chen and R.-S. Liu, Nanoscale, 2013, 5, 3547-3569.

7 F. Variola, J. B. Brunski, G. Orsini, P. T. d. Oliveira, R. Wazen and A. Nanci, Nanoscale, 2011, 3, 335-353.

8 K. Anselme, P. Davidson, A. M. Popa, M. Giazzon, M. Liley and L. Ploux, Acta Biomater., 2010, 6, 3824-3846.

9 F. Variola, F. Vetrone, L. Richert, P. Jedrzejowski, J.-H. Yi, S. Zalzal, S. Clair, A. Sarkissian, D. F. Perepichka, J. D. Wuest, F. Rosei and A. Nanci, Small, 2009, 5, 996-1006.

10 J. Hasan, R. J. Crawford and E. P. Ivanova, Trends Biotechnol., 2013, 31, 295-304.

11 G. E. Fantner, O. Rabinovych, G. Schitter, P. Thurner, J. H. Kindt, M. M. Finch, J. C. Weaver, L. S. Golde, D. E. Morse, E. A. Lipman, I. W. Rangelow and P. K. Hansma, Compos. Sci. Technol., 2006, 66, 1205-1211.

12 G. E. Fantner, T. Hassenkam, J. H. Kindt, J. C. Weaver, H. Birkedal, L. Pechenik, J. A. Cutroni, G. A. Cidade, G. D. Stucky, D. E. Morse and P. K. Hansma, Nat. Mater., 2005, 4, 612-616.

13 Y. Lin and S. Xu, J. Microsc., 2011, 241, 291-302.
14 E. Rettler, S. Hoeppener, B. W. Sigusch and U. S. Schubert, J. Mater. Chem. B, 2013, 2789-2806.

15 A. Alessandrini and P. Facci, Micron, 2012, 43, 1212-1223.

16 A. W. Coleman, A. N. Lazar, C. F. Rousseau, S. Cecillon and P. Shahgaldian, in Nanosystem characterization tools in the life sciences, ed. C. S. S. R. Kumar, Wiley-VCH, 2011.

17 G. Haugstad, Atomic Force Microscopy: understanding basic modes and advanced applications, Wiley, 2012.

18 A. Ketabchi, K. Komm, M. Miles-Rossouw, D. A. D. Cassani and F. Variola, PLoS One, 2014, 9, e92080-e92089.

19 J. Crear, K. M. Kummer and T. J. Webster, Int. J. Nanomed., 2013, 8, 995-1001.

20 L. He, P. Zhao, Q. Han, X. Wang, X. Cai, Y. Shi, L. Zhou, Y. Zhang and W. Xue, Carbon, 2013, 56, 224-234.

21 C. Huang, M. Moosmann, J. Jin, T. Heiler, S. Walheim and T. Schimmel, Beilstein J. Nanotechnol., 2012, 3, 620-628.

22 P. A. George, K. Quinn and J. J. Cooper-White, Biomaterials, 2010, 31, 641-647.

23 Y. Wang, L. Zhang, L. Sun and T. J. Webster, Int. J. Nanomed., 2013, 8, 159-166.

24 N. J. Fredin, A. H. Broderick, M. E. Buck and D. M. Lynn, Biomacromolecules, 2009, 10, 994-1003.

25 J. Brown, P. Kocher, C. S. Ramanujan, D. N. Sharp, K. Torimitsu and J. F. Ryan, Ultramicroscopy, 2013, 133, 62-66.

26 K. Suzuki, K. Kobayashi, N. Oyabu, K. Matsushige and H. Yamada, J. Chem. Phys., 2014, 140, 054704.

27 J. M. Pelto, S. P. Haimi, A. S. Siljander, S. S. Miettinen, K. M. Tappura, M. J. Higgins and G. G. Wallace, Langmuir, 2013, 29, 6099-6108.

28 E. Gongadze, D. Kabaso, S. Bauer, T. Slivnik, P. Schmuki, U. v. Rienen and A. Iglic, Int. J. Nanomed., 2011, 6, 1801-1816.

29 D. Kabaso, E. Gongadze, S. Perutkova, C. Matschegewski, V. Kralj-Iglic, U. Beck, U. v. Rienen and A. Iglic, Comput. Meth. Biomech. Biomed. Eng., 2011, 14, 469-482.

30 B. Kasemo, Surf. Sci., 2002, 500, 656-677.

31 I. O. Smith, M. J. Baumann and L. R. McCabe, J. Biomed. Mater. Res., Part A, 2004, 70, 436-441.

32 A. J. Katan and C. Dekker, Cell, 2011, 147, 979-982.

33 A. M. Whited and P. S.-H. Park, Biochim. Biophys. Acta, 2014, 1838, 56-68.

34 W. Kalle and P. Strappe, Micron, 2012, 43, 1224-1231.

35 A. Rajendran, M. Endo and H. Sugiyama, Adv. Protein Chem. Struct. Biol., 2012, 87, 5-55.

36 A. Rajendran, M. Endo and H. Sugiyama, Chem. Rev., 2014, 114, 1493-1520.

37 D. J. Muller, G. Hand, A. Engel and G. Sosinsky, EMBO J., 2002, 21, 3598-3607.

38 D. J. Muller and Y. F. Dufrene, Trends Cell Biol., 2011, 21, 461-469.

39 L. S. Dorobantu, G. G. Goss and R. E. Burrell, Micron, 2012, 43, 1312-1322.

40 F. Badique, D. R. Stamov, P. M. Davidson, M. Veuillet, G. n. Reiter, J.-N. Freund, C. M. Franz and K. Anselme, Biomaterials, 2013, 34, 2991-3001.

41 J. P. Bearinger, L. C. Dugan, L. Wu, H. Hill, A. T. Christian and J. A. Hubbell, BioTechniques, 2009, 46, 209-216. 
42 A. Raman, S. Trigueros, A. Cartagena, A. P. Z. Stevenson, M. Susilo, E. Nauman and S. A. Contera, Nat. Nanotechnol., 2011, 6, 809-814.

43 A. Cartagena and A. Raman, Biophys. J., 2014, 106, 1033-1043.

44 H. Yamashita, A. Taoka, T. Uchihashi, T. Asano, T. Ando and Y. Fukumori, J. Mol. Biol., 2012, 422, 300-309.

45 H. K. Webb, V. K. Truong, J. Hasan, R. J. Crawford and E. P. Ivanova, J. Microbiol. Methods, 2011, 86, 131-139.

46 S. Dhahri, M. Ramonda and C. Marlière, PLoS One, 2013, 8, e61663.

47 P. Bertoncini, S. L. Chevalier, S. Lavenus, P. Layrolle and G. Louarn, J. Mol. Recognit., 2012, 25, 262-269.

48 O. Marti, M. Holzwarth and M. Beil, Nanotechnology, 2008, 19, 384015-384022.

49 C. A. Rezende, L.-T. Lee and F. Galembeck, Langmuir, 2009, 25, 9938-9946.

50 K. C. Neuman and A. Nagy, Nat. Methods, 2008, 5, 491-505.

51 P. Soman, Z. Rice and C. A. Siedlecki, Micron, 2008, 39, 832-842.

52 J. J. Heinisch, P. N. Lipke, A. Beaussart, S. E. K. Chatel, V. Dupres, D. Alsteens and Y. F. Dufrêne, J. Cell Sci., 2012, 125, 4189-4195.

53 D. J. Muller and Y. F. Dufrene, Nat. Nanotechnol., 2008, 3, 261-269.

54 V. Dupres, D. Alsteens, G. Andre and Y. F. Dufrêne, Trends Microbiol., 2010, 18, 397-405.

55 L. S. Dorobantu and M. R. Gray, Scanning, 2010, 32, 74-96.

56 E. Dague, D. Alsteens, J.-P. Latgé, C. Verbelen, D. Raze, A. R. Baulard and Y. F. Dufrêne, Nano Lett., 2007, 7, 3026-3030.

57 Y. F. Dufrene, J. Bacteriol., 2002, 184, 5205.

58 C. D. Blanchette, A. Loui and T. V. Ratto, in Handbook of Molecular Force Spectroscopy, ed. A. Noy, Springer, 2005.

59 G. Chen, Y. Lv, P. Guo, C. Lin, X. Zhang, L. Yang and Z. Xu, Curr. Stem Cell Res. Ther., 2013, 8, 313-323.

60 C.-M. Cheng, R. L. S. Jr. and P. R. LeDuc, J. Biomech., 2009, 42, 187-192.

61 A. J. Engler, P. O. Humbert, B. Wehrle-Haller and V. M. Weaver, Science, 2009, 324, 208-212.

62 D. E. Discher, D. J. Mooney and P. W. Zandstra, Science, 2009, 324, 1673-1677.

63 D. E. Discher, P. Janmey and Y.-l. Wang, Science, 2005, 310, 1139-1143.

64 J. Landoulsi and V. Dupres, Phys. Chem. Chem. Phys., 2013, 15, 8429-8440.

65 C. Heu, A. Berquand, C. Elie-Caille and L. Nicod, J. Struct. Biol., 2012, 178, 1-7.

66 D. Kirmizis and S. Logothetidis, Int. J. Nanomed., 2010, 5, 137-145.

67 J. Li, D. Han and Y.-P. Zhao, Sci. Rep., 2014, 4, 3910-3920.

68 I. Medalsy, U. Hensen and D. J. Muller, Angew. Chem., Int. Ed., 2011, 50, 12103-12108.

69 Y. F. Dufrene and A. E. Pelling, Nanoscale, 2013, 5, 4094-4104.

70 L. Rizzello, R. Cingolani and P. P. Pompa, Nanomedicine, 2013, 8, 807-821.

71 S. D. Puckett, E. Taylor, T. Raimondo and T. J. Webster, Biomaterials, 2010, 31, 706-713.
72 B. Ercan, E. Taylor, E. Alpaslan and T. J. Webster, Nanotechnology, 2011, 22, 295102.

73 F. Variola, S. F. Zalzal, A. Leduc, J. Barbeau and A. Nanci, Int. J. Nanomed., 2014, 9, 2319-2325.

74 P. J. Bujalowski and A. F. Oberhauser, Methods, 2013, 60, 151-160.

75 C.-K. Lee, Y.-M. Wang, L.-S. Huang and S. Lin, Micron, 2007, 38, 446-461.

76 L. Richert, A. Boukari, S. Berner, M. Dard and J. Hemmerlé, J. Biomater. Nanobiotechnol., 2011, 2, 244-249.

77 D. J. Müller, M. Krieg, D. Alsteens and Y. F. Dufrêne, Curr. Opin. Biotechnol., 2009, 20, 4-13.

78 G. Francius, D. Alsteens, V. Dupres, S. Lebeer, S. D. Keersmaecker, J. V.-. den and Y. F. Dufrene, Nat. Protoc., 2009, 4, 939-946.

79 C. Berne, X. Ma, N. A. Licata, B. R. A. Neves, S. Setayeshgar, Y. V. Brun and B. Dragnea, J. Phys. Chem. B, 2013, 117, 10492-10503.

80 A. C. A. Wan and J. Y. Ying, Adv. Drug Delivery Rev., 2010, 62, 731-740.

81 L. Yang and T. J. Webster, Expert Opin. Drug Delivery, 2009, 6, 851-864.

82 J.-H. Jang, D. V. Schaffer and L. D. Shea, Mol. Ther., 2011, 19, 1407-1415.

83 M. Hernando-Perez, E. Pascual, M. Aznar, A. Ionel, J. R. Caston, A. Luque, J. L. Carrascosa, D. Reguera and P. J. d. Pablo, Nanoscale, 2014, 6, 2702-2709.

84 P. Ni, Z. Wang, X. Ma, N. C. Das, P. Sokol, W. Chiu, B. Dragnea, M. Hagan and C. C. Kao, J. Mol. Biol., 2012, 419, 284-300.

85 C. Carrasco, A. Luque, M. Hernando-Perez, R. Miranda, J. L. Carrascosa, P. A. Serena, M. d. Ridder, A. Raman, J. Gomez-Herrero, I. A. T. Schaap, D. Reguera and P. J. d. Pablo, Biophys. J., 2011, 100, 1100-1108.

86 C. Carrasco, A. Carreira, I. A. T. Schaap, P. A. Serena, J. Gomez-Herrero, M. G. Mateu and P. J. d. Pablo, PNAS, 2006, 103, 13706-13711.

87 R. Vaughan, B. Tragesser, P. Ni, X. Ma, B. Dragnea and C. C. Kao, J. Virol., 2014, 88, 6483-6491.

88 J. Helenius, C.-P. Heisenberg, H. E. Gaub and D. J. Muller, J. Cell Sci., 2008, 121, 1785-1791.

89 E. Lamers, J. t. Riet, M. Domanski, R. Luttge, C. G. Figdor, J. G. E. Gardeniers, X. F. Walboomers and J. A. Jansen, Eur. Cells Mater., 2012, 23, 182-194.

90 T. Bornschlögl, S. p. Romero, C. L. Vestergaard, J.-F. o. Joanny, G. T. V. Nhieu and P. Bassereau, PNAS, 2013, 110, 18928-18933.

91 G. Zeng, T. Müller and R. L. Meyer, Langmuir, 2014, 30, 4019-4025.

92 C.-C. Wu, D. N. Reinhoudt, C. Otto, V. Subramaniam and a. A. H. Velders, Small, 2011, 7, 989-1002.

93 G. Arrabito, S. Reisewitz, L. Dehmelt, P. I. Bastiaens, B. Pignataro, H. Schroeder and C. M. Niemeyer, Small, 2013, 9, 4243-4249.

94 J. E. Bear and J. M. Haugh, Curr. Opin. Cell Biol., 2014, 30, 74-82. 
95 L. S. Wong, C. V. Karthikeyan, D. J. Eichelsdoerfer, J. Micklefield and C. A. Mirkin, Nanoscale, 2012, 4, 659-666.

96 N. P. Westcott, W. Luo and M. Yousaf, J. Colloid Interface Sci., 2014, 43, 207-213.

97 A. Boisen, S. Dohn, S. S. Keller, S. Schmid and M. Tenje, Rep. Prog. Phys., 2011, 74, 036101-036131.

98 C. Steffens, F. L. Leite, C. C. Bueno, A. Manzoli and P. S. D. P. Herrmann, Sensors, 2012, 12, 8278-8300.

99 S. Hsieh, S.-L. Hsieh, C.-w. Hsieh, P.-C. Lin and C.-H. Wu, J. Anal. Methods Chem., 2013, 2013, 1-5.

100 S. Aghayee, C. Benadiba, J. Notz, S. Kasas and D. a. G. Longo, J. Mol. Recognit., 2013, 26, 590-595.

101 N. Jung, H. Seo, D. Lee, C. Y. Ryu and S. Jeon, Macromolecules, 2008, 41, 6873-6875.

102 H. C. McCaig, E. Myers, N. S. Lewis and M. L. Roukes, Nano Lett., 2014, 14, 3728-3732.

103 K. S. Hwang, S.-M. Lee, S. K. Kim, J. H. Lee and a. T. S. Kim, Annu. Rev. Anal. Chem., 2009, 2, 77-98.

104 C. J. Fee, Methods Mol. Biol., 2013, 996, 287-312.

105 C. J. Fee, Methods Mol. Biol., 2013, 996, 313-322.

106 N. Kodera, D. Yamamoto, R. Ishikawa and T. Ando, Nature, 2010, 468, 72-77.

107 I. Chourpa, F. H. Lei, P. Dubois, M. Manfait and G. D. Sockalingum, Chem. Soc. Rev., 2008, 37, 993-1000.

108 T. Schmid, L. Opilik, C. Blum and R. Zenobi, Angew. Chem., Int. Ed., 2013, 52, 5940-5954.

109 C. Blum, T. Schmid, L. Opilik, S. Weidmann, S. R. Fagerer and R. Zenobi, J. Raman Spectrosc., 2012, 43, 1895-1904.

110 B. Kainz, E. A. Oprzeska-Zingrebe and J. L. Toca-Herrera, Biotechnol. J., 2014, 9, 51-60.

111 B. Harke, J. V. Chacko, H. Haschke, C. Canale and A. Diaspro, Opt. Nanosc., 2012, 1, 1-6.

112 K.-A. D. Walker, S. H. Doak and P. R. Dunstan, Ultramicroscopy, 2011, 111, 1200-1205.

113 K.-A. D. Walker, C. Morgan, S. H. Doak and P. R. Dunstan, PLoS One, 2012, 7, e31592.
114 H. A. Huckabay, K. P. Armendariz, W. H. Newhart, S. M. Wildgen and R. C. Dunn, in Nanoimaging: methods and protocols, ed. A. A. Sousa and M. J. Kruhlak, Springer, 2013.

115 P. Hinterdorfer, M. F. Garcia-Parajo and Y. F. Dufrene, Acc. Chem. Res., 2012, 45, 327-336.

116 K. Klein, A. M. Gigler, T. Aschenbrenner, R. Monetti, W. Bunk, F. Jamitzky, G. Morfill, R. W. Stark and J. Schlegel, Biophys. J., 2012, 102, 360-368.

117 M. Knauer, N. P. Ivleva, X. Liu, R. Niessner and C. Haisch, Anal. Chem., 2010, 82, 2766-2772.

118 M. Fries and A. Steele, in Confocal Raman Microscopy, ed. T. Dieing, O. Hollricher and J. Toporski, Springer, 2010.

119 K. Wormuth, in Confocal Raman Microscopy, ed. T. Dieing, O. Hollricher and J. Toporski, Springer, 2010.

120 U. Schmidt, J. Müller and J. Koenen, in Confocal Raman Microscopy, ed. T. Dieing, O. Hollricher and J. Toporski, Springer, 2010.

121 T. Wermelinger and R. Spolenak, in Confocal Raman Microscopy, ed. T. Dieing, O. Hollricher and J. Toporski, Springer, 2010.

122 R. Treffer, R. Bohme, T. Deckert-Gaudig, K. Lau, S. Tiede, X. Lin and V. Deckert, Biochem. Soc. Trans., 2012, 40, 609-614.

123 S. Najjar, D. Talaga, L. Schue, Y. Coffinier, S. Szunerits, R. Boukherroub, L. Servant, V. Rodriguez and S. Bonhommeau, J. Phys. Chem. C, 2014, 118, 1174-1181.

124 A. Tarun, N. Hayazawa and S. Kawata, Anal. Bioanal. Chem., 2009, 394, 1775-1785.

125 E. A. Pozzi, M. D. Sonntag, N. Jiang, J. M. Klingsporn, M. C. Hersam and R. P. V. Duyne, ACS Nano, 2013, 7, 885-888.

126 T. Deckert-Gaudig and V. Deckert, Curr. Opin. Chem. Biol., 2011, 15, 719-724.

127 K. F. Domke and B. Pettinger, ChemPhysChem, 2010, 11, 1365-1373.

128 A. P. D. Elfick, A. R. Downes and R. Mouras, Anal. Bioanal. Chem., 2010, 396, 45-52. 